\title{
Electronic Density-Matrix Algorithm for Nonadiabatic Couplings in Molecular Dynamics Simulations
}

\author{
M. TOMMASINI, ${ }^{1}$ V. CHERNYAK, ${ }^{2}$ S. MUKAMEL ${ }^{2}$ \\ ${ }^{1}$ Dipartimento di Chimica Industriale e Ingegneria Chimica, Politecnico di Milano, Piazza Leonardo da \\ Vinci 32, 20133 Milano, Italy \\ ${ }^{2}$ Department of Chemistry, University of Rochester, P. O. RC Box 270216 Rochester, NY 14627-0216
}

Received 25 February 2001; accepted 2 March 2001

\begin{abstract}
Closed expressions for nonadiabatic couplings are derived using the collective electronic oscillators (CEO) algorithm based on the time-dependent Hartree-Fock equations. Analytic derivatives allow the calculation of transition density matrices and potential surfaces at arbitrary nuclear geometries using a molecular dynamics trajectory that only requires a CEO calculation at a single configuration. 두 2001 John Wiley \& Sons, Inc. Int J Quantum Chem 00: 1-14, 2001
\end{abstract}

\section{Key words:}

\section{Introduction}

$\mathbf{T}$ he simulation of photoinduced processes in molecules requires the computation of electronic potential surfaces and nonadiabatic couplings between them $[1,2]$. The collective electronic oscillators approach (CEO) provides an inexpensive algorithm for computing adiabatic potential surfaces $[3,4]$. This constitutes a major computational saving since a quantity which carries much less information than the many electron wavefunction of the excited states, namely the transition density ma-

Correspondence to: S. Mukamel; e-mail: mukamel@chem. rochester.edu.

Contract grant sponsor: National Science Foundation and the Petroleum Research Fund, American Chemical Society. trix between ground and excited states, is computed instead [5]. These matrices further represent collective oscillators or electronic normal modes that describe the time evolution of the density matrix of the system under the effects of an external perturbation. The CEO eigenvalue equation can be written as

$$
L \xi_{k}=\Omega_{k} \xi_{k}
$$

$L$ is the effective TDHF Liouville operator for the molecule, whose spectrum describes the electronic excitations of the system. The atomic orbital representation of the oscillators $\xi_{k}$ provides a clear characterization of molecular excitations in real space [6]. The eigenvectors $\xi_{k}$ are normalized according to: ${ }^{1}\left(\xi_{k}, \xi_{k}\right)=\operatorname{Tr}\left(\bar{\rho}\left[\xi_{k}^{\dagger}, \xi_{k}\right]\right)=1$, where $\bar{\rho}$ is

\footnotetext{
${ }^{1}$ The scalar product introduced here to normalize the eigenvectors $\xi_{k}$ is discussed in Appendix A.
} 
the Hartree-Fock ground state density matrix. The sum of the excitation energy $\Omega_{k}(\mathbf{R})$ and the ground state energy $\varepsilon_{0}(\mathbf{R})$ at different nuclear configurations $\mathbf{R}$ gives the excited state adiabatic potential surface $\varepsilon_{k}(\mathbf{R})$. These quantities are sufficient to describe the nuclear dynamics of a molecule in regions of the nuclear space far away from avoided crossings or conical intersections. However, many interesting photoinduced processes involve the transition between different potential surfaces in the vicinity of an avoided crossing or conical intersection [7]. An example of such a process is the cis to trans photoisomerization of rhodopsin which is the elementary process in vision [8-10]. Under these circumstances it is crucial to include the nonadiabatic couplings between different potential surfaces in order to properly describe the joint electronic and nuclear dynamics, and to predict the branching ratios among different reaction pathways.

The aim of this work is to derive closed expressions for the nonadiabatic couplings (NAC) between any pair of electronic states in the CEO framework. The close connection between the NAC and the derivatives of the density matrices further provides the formal solution of the equations proposed in [3] in terms of CEO eigenvectors and NAC.

The first-order nonadiabatic couplings are conveniently defined in terms of a vector potential in nuclear space [11]

$$
A_{i k}^{x} \equiv-\left\langle i\left|\frac{\partial}{\partial x}\right| k\right\rangle
$$

where $\langle i|$ and $\langle k|$ are two adiabatic electronic states and $x$ is a generic nuclear coordinate. In Appendix B we use perturbation theory to express the NAC in the form

$$
A_{i k}^{x}=\frac{\left\langle i\left|\hat{h}^{x}\right| k\right\rangle}{\varepsilon_{i}-\varepsilon_{k}} ; \quad i \neq k
$$

where $\hat{h}^{x}$ is the derivative of the molecular Hamiltonian $\hat{H}$ with respect to a nuclear coordinate $x .^{2}$ This expression is convenient for numerical computations. Introducing a basis set, with the corresponding fermionic creation and annihilation operators $c_{n}^{\dagger}$ and $c_{n}$, we have

$$
A_{i k}^{x}=\frac{\operatorname{Tr}\left(\rho_{i k} h^{x}\right)}{\Omega_{i}-\Omega_{k}} ; \quad i \neq k .
$$

$\left(\rho_{i k}\right)_{n m}=\left\langle i\left|c_{n}^{\dagger} c_{m}\right| k\right\rangle$ denotes the one-electron transition density matrix and the excited state energy

\footnotetext{
${ }^{2}$ Throughout this work we use the short-hand notation and denote the derivative with respect to a nuclear coordinate $x$ by $x$ superscript.
}

has been rewritten in terms of the ground state plus the excitation energy $\varepsilon_{k}=\varepsilon_{0}+\Omega_{k}$. The matrix $h^{x}$ represents the operator $\hat{h}^{x}$ in the chosen basis set $\hat{h}^{x}=\Sigma_{n m} h_{n m}^{x} c_{n}^{\dagger} c_{m}$. The expression for NAC involving the ground state is even simpler and only requires the knowledge of $\left(\rho_{i 0}\right)_{n m}=\left\langle i\left|c_{n}^{\dagger} c_{m}\right| 0\right\rangle \equiv\left(\xi_{i}\right)_{n m}$ and $\left(\rho_{0 i}\right)_{n m}=\left\langle 0\left|c_{n}^{\dagger} c_{m}\right| i\right\rangle \equiv\left(\xi_{i}^{\dagger}\right)_{n m}$

$$
A_{i 0}^{x}=\frac{\operatorname{Tr}\left(\xi_{i} h^{x}\right)}{\Omega_{i}} ; \quad A_{0 i}^{x}=\frac{\operatorname{Tr}\left(\xi_{i}^{\dagger} h^{x}\right)}{\Omega_{i}} .
$$

The matrices $\left\{\xi_{i}\right\}$ are obtained from the collective electronic oscillators algorithm [6,12]. The transition density matrices between excited states, $\rho_{i k}$, can be also expressed in terms of the CEO [13]. To use Eqs. (4) and (5) we need to express the operator $\hat{h}^{x}$ in terms of the derivative of the matrix elements of the molecular Hamiltonian. In Appendix $C$ we show that the matrix elements of the operator $\hat{h}^{x}$ can be expressed in an atomic orbital representation as

$$
h^{x}=t^{x}+V^{x} \bar{\rho}
$$

where $t^{x}$ is the derivative of the matrix elements for the one-electron part of the Hamiltonian and $V^{x}$ is the derivative of the electron-electron interaction operator. The operator $\hat{h}^{x}$ represents the vibronic coupling of the molecular Hamiltonian and it is the source of the nonadiabatic coupling. These results will be discussed in the coming sections. The second section presents the derivatives of the ground state density matrix and of the CEO transition density matrices. In the third section we present the equations for classical molecular dynamics on excited state hypersurfaces using the CEO/TDHF approach. An analytic expression for the derivatives of the density matrices is also derived. In the fourth section we discuss some general properties of nonadiabatic couplings resulting from the translational symmetry of the electronic Hamiltonian when expressed using atomic orbitals centered on the nuclei. Similar properties are derived also for the density matrices. In the fifth section we discuss the role of nonadiabatic couplings in determining a contribution to vibrational infrared absorption intensities. Finally, the Appendixes present details of the derivations and the CEO algorithm.

\section{Analytic Derivatives}

By solving the Hartree-Fock equation for the density matrix at various nuclear configurations 
and the CEO eigenvalue problem [Eq. (1)] it is possible to construct the entire adiabatic potential energy surfaces. The nonadiabatic couplings at each nuclear configuration can then be computed from the knowledge of the CEO transition matrices and the transition energies, using Eqs. (4) and (5). A different strategy for achieving the same goal is to use the analytic derivatives of all quantities with respect to the nuclear coordinates. It is then possible in principle to carry out the full Hartree-Fock/CEO calculation only once, at some chosen initial configuration. The analytic derivatives then allow us to compute the quantities of interest at all configurations by running a trajectory; we can thus generate the density matrices on the fly while avoiding a new Hartree-Fock SCF or a new CEO computation in the displaced nuclear configuration. Such strategy is reminiscent of Car-Parrinello simulations [14]. In this section we provide closed expressions for these derivatives. Numerical considerations should determine which strategy is to be preferred: repeating the CEO or using the derivatives. Making use of these results for the NAC it is possible to compute the derivative of the ground state density matrix and the derivative of the CEO eigenvectors.

A general relationship exists between the derivative of the density matrices and the NAC (see Appendix D)

$$
\rho_{i j}^{x}=\sum_{k \neq i} A_{i k}^{x} \rho_{k j}-\sum_{k \neq j} \rho_{i k} A_{k j}^{x} .
$$

In particular we have for the ground state density matrix

$$
\bar{\rho}^{x}=\sum_{j>0} A_{0 j}^{x} \xi_{j}-A_{j 0}^{x} \xi_{j}^{\dagger} .
$$

Equation (8) shows that the derivative of the ground state density matrix is given by a weighted sum over the CEO modes, where the coefficients are given by the nonadiabatic couplings between the ground and the various excited states. These nonadiabatic couplings can be computed analytically using Eq. (5) once the CEO eigenvectors are known by solving Eq. (1). A closed expression may also be derived for the derivative of the transition density matrix $\xi_{i}$ (see the third section)

$$
\begin{aligned}
\xi_{i}^{x}= & \sum_{k>0 ; k<0 ; k \neq i} \frac{\xi_{k}\left(\xi_{k}, D_{i}^{x}\right)}{\Omega_{k}-\Omega_{i}}+(1-2 \bar{\rho})\left[\xi_{i}, \bar{\rho}^{x}\right]_{+} ; \\
D_{i}^{x}= & {\left[\left[C_{i}^{x}, \bar{\rho}\right], \bar{\rho}\right] ; } \\
C_{i}^{x}= & -\left[h^{x}, \xi_{i}\right]-\left[V^{x} \xi_{i}, \bar{\rho}\right]-\left[V \bar{\rho}^{x}, \xi_{i}\right] \\
& -\left[V \xi_{i}, \bar{\rho}^{x}\right]+\Omega_{i}^{x} \xi_{i} ;
\end{aligned}
$$

$$
\begin{aligned}
\Omega_{i}^{x} & =\left(\xi_{i}, L^{x} \xi_{i}\right) ; \\
L^{x} \zeta & =\left[F(\bar{\rho})^{x}, \zeta\right]+\left[V^{x} \zeta, \bar{\rho}\right]+\left[V \zeta, \bar{\rho}^{x}\right] .
\end{aligned}
$$

Here and in the remainder of this paper $\zeta$ denotes a matrix representing an arbitrary single electron operator: Eq. (9) shows the action of superoperators in Liouville space. The scalar product $\left(\zeta_{1}, \zeta_{2}\right)$ in Eq. (9) is defined as

$$
\left(\zeta_{1}, \zeta_{2}\right)=\left(\zeta_{1} \mid \zeta_{2}\right)=\operatorname{Tr}\left(\bar{\rho}\left[\zeta_{1}^{\dagger}, \zeta_{2}\right]\right)
$$

With respect to this scalar product the Liouville operator $L \zeta=[F(\bar{\rho}), \zeta]+[V \zeta, \bar{\rho}]$ of Eq. (1) is Hermitian: $\left(L \eta_{1}, \eta_{2}\right)=\left(\eta_{1}, L \eta_{2}\right)$ (see Appendix A). $F(\bar{\rho})^{x}$ is the derivative of the Fock matrix corresponding to the ground state density matrix $\bar{\rho}$ (see Appendix $\mathrm{H}$ ).

\section{Classical Nuclear Dynamics Simulations}

Many processes and spectroscopic properties may be adequately simulated by running classical trajectories on excited state surfaces. However, the classical description breaks down at curve crossing points where a quantum wave packet calculation is required [7]. In [3] the Hartree-Fock equation for the ground state and the CEO algorithm for the TDHF were differentiated with respect to nuclear coordinates in order to get closed equations that can describe the molecular dynamics on excited state hypersurfaces. We recast here the equations of [3] using a somewhat different notation and adopting a different point of view which underscores the interesting role of the nonadiabatic couplings. We consider both electronic and nuclear degrees of freedom and write equations of motion for the variables of interest along the classical trajectory of the nuclei. The state of the molecule along this trajectory at a given time $\tau$ will be defined by the following quantities: the nuclear coordinates $\mathbf{x}(\tau)$, the Hartree-Fock ground state density matrix $\bar{\rho}(\mathbf{x}(\tau))$, and the CEO transition matrix $\xi_{i}(\mathbf{x}(\tau))$. On a given $i$ th excited state surface these satisfy the equations of motion:

$$
\begin{aligned}
m_{\alpha} \ddot{x}_{\alpha} & =-\frac{d}{d x_{\alpha}}\left[E_{0}(\mathbf{x})+\Omega_{i}(\mathbf{x})\right], \\
\frac{\partial \bar{\rho}}{\partial \tau} & =\sum_{\alpha} \bar{\rho}^{x_{\alpha}} \dot{x}_{\alpha}, \\
\frac{\partial \xi_{i}}{\partial \tau} & =\sum_{\alpha} \xi_{i}^{x_{\alpha}} \dot{x}_{\alpha} .
\end{aligned}
$$

The ground state density matrix $\bar{\rho}$ satisfies for each nuclear configuration $\mathbf{x}(\tau)$ the self consistent 
Hartree-Fock equation $[F, \bar{\rho}]=0$, where $F$ is the Fock operator which depends on $\rho$. In the classical equation of motion for the nuclear coordinates [the first of Eqs. (11)] we used the sum of the ground state energy and the excitation energy to generate the excited state surface

$$
E_{i}(\mathbf{x})=E_{0}(\mathbf{x})+\Omega_{i}(\mathbf{x})=2 \operatorname{Tr}\left[\bar{\rho} t+\frac{1}{2} \bar{\rho} V \bar{\rho}\right]+\Omega_{i}(\mathbf{x}) .
$$

The excitation energy $\Omega_{i}$ at each nuclear configuration $\mathbf{x}$ is the eigenvalue of the CEO equation, Eq. (1). The necessary excited state energy gradient can be computed as

$$
\begin{aligned}
\frac{d}{d x_{\alpha}}\left[E_{0}(\mathbf{x})+\Omega_{i}(\mathbf{x})\right]=2 \operatorname{Tr}\left[\bar{\rho} t^{x_{\alpha}}+\right. & \left.\frac{1}{2} \bar{\rho} V^{x_{\alpha}} \bar{\rho}\right] \\
& +\left(\xi_{i}, L^{x_{\alpha}} \xi_{i}\right) .
\end{aligned}
$$

Here the ground state energy gradient has been expressed using the derivatives of the one-electron parameters $t^{x_{\alpha}}$ and two-electron parameters $V^{x_{\alpha}}$. The derivative of the Liouville operator with respect to a nuclear coordinate $L^{x_{\alpha}}$ can be expanded as the last of Eqs. (9). Equations (11) together with the analytic expressions for the derivatives of the density matrices along the nuclear coordinates, e.g., $\bar{\rho}^{x_{\alpha}}$ and $\xi_{i}^{x_{\alpha}}$, Eqs. (8) and (9), are closed.

These equations can be solved by computing the CEO spectrum at a given initial point and then by propagating the density matrices along the classical trajectory. ${ }^{3}$ This follows the spirit of Car-Parrinello simulations on the ground state [14].

We next show how to solve the two equations for the derivatives of the density matrices in terms of the CEO eigenvectors and the NAC.

The derivative of the ground state density matrix satisfies the following linear equation (see Appendix C, Eq. (72) and Appendix F):

$$
L \bar{\rho}^{x}=\left[\bar{\rho}, h^{x}\right] .
$$

Using the perturbative expression for $\bar{\rho}^{x}$, Eq. (79), and the expressions for the NAC involving the ground state, Eq. (5), we can express the solution of Eq. (14) as follows: ${ }^{4}$

$$
\bar{\rho}^{x}=\sum_{j>0} A_{0 j}^{x} \xi_{j}-A_{j 0}^{x} \xi_{j}^{\dagger}
$$

\footnotetext{
${ }^{3}$ For example, if we consider a given small displacement $\delta$ of a given nuclear coordinate $x$, we can propagate the density matrix $\bar{\rho}$ from $x$ to $x+\delta$ through the use of the analytic derivative $\bar{\rho}^{x}$, e.g., $\bar{\rho}(x+\delta) \approx \bar{\rho}(x)+\bar{\rho}^{x}(x) \delta$.

${ }^{4}$ The Hermiticity of $\bar{\rho}^{x}$ is assured by the following property of the NAC: $\left(A_{0 j}^{x}\right)^{*}=-A_{j 0}^{x} ;\left(A_{j 0}^{x}\right)^{*}=-A_{0 j}^{x}$. This property can be directly derived from the perturbative expression of the NAC, Eq. (3). Note that $\hat{h}^{x}$ is Hermitian.
}

It is then possible to compute $\bar{\rho}^{x}$ once the NAC involving the ground state are obtained through the knowledge of the CEO eigenmodes and the matrix elements of $\hat{h}^{x}$ [Eq. (6)]. On the other hand, using Eq. (15) and the algebraic properties of the CEO eigenvectors, we can express the NAC involving the ground state as scalar products of $p^{x}$ with the CEO eigenvectors:

$$
\begin{aligned}
& A_{0 j}^{x}=\left(\xi_{j}, \bar{\rho}^{x}\right) ; \\
& A_{j 0}^{x}=\left(\xi_{j}^{\dagger}, \bar{\rho}^{x}\right) .
\end{aligned}
$$

This alternative to Eq. (5) for computing the NAC is particularly useful when the derivative of the density matrix is already available (e.g., $\bar{\rho}^{x}$ may be easily obtained from a finite differences HartreeFock SCF computation).

We next turn to the derivative of the CEO eigenmodes $\xi_{i}^{x}$. Applying $\partial / \partial x$ to the equation $L \xi_{i}=\Omega_{i} \xi_{i}$ gives [3]

$$
\left(L-\Omega_{i}\right) \xi_{i}^{x}=-\left(L^{x}-\Omega_{i}^{x}\right) \xi_{i} .
$$

The right-hand side of Eq. (17) is assumed to be known and will be denoted $C_{i}^{x}$. $C_{i}^{x}$ can be directly computed once the equation for $\bar{\rho}^{x}$, Eq. (14), has been solved and the derivative of the CEO eigenvalue $\Omega_{i}^{x}=\left(\xi_{i}, L^{x} \xi_{i}\right)$ has been evaluated. More explicitly, expanding the derivative of the Liouville operator $^{5}$ we get

$$
\begin{aligned}
& \left(L-\Omega_{i}\right) \xi_{i}^{x}=C_{i}^{x} ; \\
& C_{i}^{x}=-\left[h^{x}, \xi_{i}\right]-\left[V^{x} \xi_{i}, \bar{\rho}\right]-\left[V \bar{\rho}^{x}, \xi_{i}\right] \\
& \quad-\left[V \xi_{i}, \bar{\rho}^{x}\right]+\Omega_{i}^{x} \xi_{i} .
\end{aligned}
$$

In Appendix $C$ we show that it is possible to expand the derivative of the ground state density matrix in the $\mathrm{CEO}$ eigenmodes $\xi_{i}$. The reason is that the derivative of the density matrix belongs to the particlehole space and the eigenmodes of $L$ form a complete basis set for that space (see Appendix E). On the other hand $\xi_{i}^{x}$ does not belong to the particle-hole space spanned by the set of the CEO eigenvectors $\left\{\xi_{i}\right\}$. This is because the particle-hole spaces associated with different density matrices $\bar{\rho}$ are in general different. Nevertheless, if the two density matrices are close enough (as when evaluating the derivative of $\xi_{i}$ ) it is possible to express the part of $\xi_{i}^{x}$ lying outside the particle-hole space associated with $\bar{\rho}$, in terms of the derivative of the density matrix $\bar{\rho}^{x}$ (Appendix G). $\xi_{i}^{x}$ may thus be expressed as a sum of an interband $\gamma_{i}^{x}$ and an intraband $\varphi_{i}^{x}$ component

$$
\xi_{i}^{x}=\gamma_{i}^{x}+\varphi_{i}^{x} .
$$

\footnotetext{
${ }^{5}$ See, e.g., the last of Eqs. (9) and Appendix H.
} 
These two components are evaluated in Appendix G. $\varphi_{i}^{x}$ is obtained by making use of Eq. (108): $\varphi_{i}^{x}=(1-2 \bar{\rho})\left[\xi_{i}, \bar{\rho}^{x}\right]_{+} \cdot \gamma_{i}^{x}$ is given by Eq. (114). Thus we finally obtain the analytic expression of the derivative of the CEO transition density matrix (the prime denotes that the $i$ th mode is excluded from the sum)

$$
\xi_{i}^{x}=\sum_{k>0 ; k<0} \frac{\xi_{k}\left(\xi_{k}, D_{i}^{x}\right)}{\Omega_{k}-\Omega_{i}}+(1-2 \bar{\rho})\left[\xi_{i}, \bar{\rho}^{x}\right]_{+^{\prime}}
$$

where

$$
D_{i}^{x}=\left[\left[C_{i}^{x}, \bar{\rho}\right], \bar{\rho}\right]
$$

\section{Sum Rules For Nonadiabatic Couplings and Transition Density Matrices}

We first consider a sum rule for HellmanFeynman forces. The invariance of the electronic energy $\varepsilon_{k}(\mathbf{R})$ with respect to a rigid translation $\mathbf{T}$ of nuclear coordinates $\{\mathbf{R}\}$, results in the following sum rule:

$$
\sum_{\lambda}\left\langle k(\mathbf{R})\left|\hat{h}^{x \lambda}(\mathbf{R})\right| k(\mathbf{R})\right\rangle=0,
$$

where we have defined $\hat{h}^{x \lambda}(\mathbf{R}) \equiv \partial \hat{H}(\mathbf{R}) / \partial x_{\lambda}$. This simply implies that the sum of the HellmanFeynman forces acting on the nuclei is zero, both for the ground or for an excited state. The sum is extended over all $N$ atoms, and the same equation can be written for the $y$ and the $z$ derivatives. A rigid translation $\mathbf{T}$ acting on the nuclear space cannot change the electronic energy, i.e., $\varepsilon_{k}(\mathbf{R}+\mathbf{T})=\varepsilon_{k}(\mathbf{R})$. In particular for an infinitesimal translation $\Delta$ along the $x$ axis, we have

$$
\varepsilon_{k}(\mathbf{R}+\Delta)=\varepsilon_{k}(\mathbf{R})
$$

expanding $\varepsilon_{k}(\mathbf{R}+\Delta)$ to first order in $\Delta$, results in

$$
\varepsilon_{k}(\mathbf{R})+\sum_{\lambda} \Delta \frac{\partial \varepsilon_{k}}{\partial x_{\lambda}}(\mathbf{R})=\varepsilon_{k}(\mathbf{R})
$$

which immediately gives

$$
\sum_{\lambda} \frac{\partial \varepsilon_{k}}{\partial x_{\lambda}}(\mathbf{R})=0 .
$$

Making use of the Hellmann-Feynman theorem [see Appendix B, Eq. (66)], we obtain

$$
\sum_{\lambda} \frac{\partial \varepsilon_{k}}{\partial x_{\lambda}}(\mathbf{R})=\sum_{\lambda}\left\langle k(\mathbf{R})\left|\hat{h}^{x_{\lambda}}(\mathbf{R})\right| k(\mathbf{R})\right\rangle=0 .
$$

Sum rules for nonadiabatic couplings and density matrix derivatives can be derived in a similar way. The integrals over atomic basis functions that enter the definition of the Hamiltonian in the atomic orbital basis set must depend only on the relative positions of the atoms, thus $t_{i j}$ and $(i j \mid k l)$ are invariant to an overall translation of the molecule. Through the same reasoning used for $\varepsilon_{k}$ we immediately get

$$
\sum_{\lambda} t_{i j}^{x_{\lambda}}=0 ; \quad \sum_{\lambda}(i j \mid k l)^{x \lambda}=0 .
$$

This yields the following sum rules for the derivatives of the Coulomb and exchange operator, the Fock matrix ${ }^{6}$ and $h^{x}$

$$
\begin{aligned}
\sum_{\lambda} J^{x \lambda} \bar{\rho}=\sum_{\lambda} K^{x \lambda} \bar{\rho} & =0 ; & & \sum_{\lambda} V^{x \lambda} \bar{\rho}=0 ; \\
\sum_{\lambda} F^{x \lambda} & =0 ; & & \sum_{\lambda} h^{x \lambda}=0 .
\end{aligned}
$$

In the atomic orbital representation the NAC Eq. (4) assumes the form

$$
A_{i k}^{x}=\frac{\sum_{n m}\left(\rho_{i k}\right)_{n m}\left(h^{x}\right)_{n m}}{\Omega_{i}-\Omega_{k}}: \quad i \neq k .
$$

If we express the sum rule for $h^{x}$ in the atomic orbital representation, Eq. (28), we obtain the following sum rule for the NAC:

$$
\sum_{\lambda} A_{i k}^{x \lambda}=\sum_{\lambda} A_{i k}^{y \lambda}=\sum_{\lambda} A_{i k}^{z \lambda}=0 .
$$

This relationship can be used as a consistency check for the computation of NAC.

Let us turn now to the derivative of the oneparticle transition density matrix connecting two electronic levels $|i\rangle$ and $|j\rangle$ in the atomic orbital representation $\left(\rho_{i j}^{x}\right)_{n m} \equiv(\partial / \partial x)\left\langle i\left|c_{n}^{\dagger} c_{m}\right| j\right\rangle$. Combining the above sum rule for the NAC with Eq. (7) we immediately get the following sum rule for the transition density matrices:

$$
\sum_{\lambda=1}^{N} \rho_{i j}^{x \lambda}=\sum_{\lambda=1}^{N} \rho_{i j}^{y \lambda}=\sum_{\lambda=1}^{N} \rho_{i j}^{z \lambda}=0 .
$$

As special cases of Eq. (31) we have for the derivatives of the ground state density matrix and the CEO eigenvectors $\left\{\xi_{i}\right\}$

$$
\begin{aligned}
& \sum_{\lambda=1}^{N} \bar{\rho}^{x \lambda}=\sum_{\lambda=1}^{N} \bar{\rho}^{y \lambda}=\sum_{\lambda=1}^{N} \bar{\rho}^{z \lambda}=0 ; \\
& \sum_{\lambda=1}^{N} \xi_{i}^{x \lambda}=\sum_{\lambda=1}^{N} \xi_{i}^{y \lambda}=\sum_{\lambda=1}^{N} \xi_{i}^{z \lambda}=0 .
\end{aligned}
$$

\footnotetext{
${ }^{6}$ The sum rule for the Fock matrix holds provided that a similar sum rule exist for the density matrix. This is introduced later.
} 


\section{Discussion}

Infrared intensities may be related to the electron mobility within molecules. They also enter the expressions for vibrational hyperpolarizabilities [16]. The contribution of the electronic charge to the molecular dipole moment can be written in terms of the ground state density matrix $\bar{\rho}$ and the dipole operator $\mu^{s}$ (the superscript $s$ denotes the sth Cartesian component)

$$
M^{s}=\operatorname{Tr}\left(\mu^{s} \bar{\rho}\right) .
$$

The infrared intensity is quadratic in the derivatives of the dipole moment with respect to nuclear coordinates [17]. The derivative of $M^{s}$ with respect to a generic nuclear coordinate $x$ is

$$
\frac{\partial M}{\partial x}=\operatorname{Tr}\left(\frac{\partial \mu^{s}}{\partial x} \bar{\rho}\right)+\operatorname{Tr}\left(\mu^{s} \frac{\partial \bar{\rho}}{\partial x}\right) .
$$

The first term is known as the charge contribution to the infrared intensity. It represents the contribution to the change in the dipole moment arising from the displacement along the vibrational coordinate $x$ of fixed charges associated with the atomic orbital basis set. The second term, known as the flux contribution, reflects the redistribution of electronic charges upon nuclear motion. This term can be directly related to the nonadiabatic coupling vector potential between the ground and excited states $A_{0 j}^{x}$. To show this we recall the expansion of the density matrix derivative in the CEO eigenmodes, Eq. (15), i.e., $\bar{\rho}^{x}=\sum_{j>0} A_{0 j}^{x} \xi_{j}-A_{j 0}^{x} \xi_{j}^{\dagger}$. Substitution of Eq. (15) in Eq. (34) gives

$$
\frac{\partial M^{s}}{\partial x}=\operatorname{Tr}\left(\frac{\partial \mu^{s}}{\partial x} \bar{\rho}\right)+\sum_{j>0} A_{0 j}^{x} \operatorname{Tr}\left(\mu^{s} \xi_{j}\right)-A_{j 0}^{x} \operatorname{Tr}\left(\mu^{s} \xi_{j}^{\dagger}\right) \text {. }
$$

The infrared flux is thus given by a sum of the transition dipoles between the ground state and the manifold of excited states weighted by the nonadiabatic couplings between ground and excited states. For conjugated molecules, such as Carotenoids, in which the ECC infrared bands are dominated by contributions only from few excited states, the experimentally accessible infrared intensities [18, 19] could be used to estimate the magnitude of the nonadiabatic couplings. Making use of the NAC sum rule it is possible to show that the sum of the infrared fluxes with respect to the Cartesian coordinate $x_{\lambda}, y_{\lambda}$, or $z_{\lambda}$ must vanish. This is consistent with the charge conservation principle from which the sum rule on infrared fluxes is usually derived.
We further note that the first-order nonadiabatic couplings may be used to compute also the secondorder nonadiabatic couplings. This can be done as follows:

$$
A_{i j}^{x y} \equiv\left\langle i\left|\frac{\partial^{2}}{\partial x \partial y}\right| j\right\rangle=\left\langle i\left|\frac{\partial}{\partial x} \hat{i} \frac{\partial}{\partial y}\right| j\right\rangle .
$$

By considering the resolution of the identity $\hat{1}=$ $\sum_{k}|k\rangle\langle k|$, we have

$$
A_{i j}^{x y}=\sum_{k}\left\langle i\left|\frac{\partial}{\partial x}\right| k\right\rangle\left\langle k\left|\frac{\partial}{\partial y}\right| j\right\rangle .
$$

This gives

$$
A_{i j}^{x y}=\sum_{k} A_{i k}^{x} A_{k j}^{y}
$$

\section{Appendix A: The CEO Algorithm}

We introduce here the basic quantities required for the CEO algorithm. The molecular Hamiltonian can be written using second quantization as follows $[20,21]$ :

$$
\begin{aligned}
\hat{H}=\sum_{i j} t_{i j} c_{i}^{\dagger} c_{j}+\frac{1}{2} \sum_{i j k l}\langle i j \mid k l\rangle c_{i}^{\dagger} c_{j}^{\dagger} c_{l} c_{k} & \\
& -\sum_{s=x, y, z} \mathcal{E}^{s}(t) \sum_{i j} \mu_{i j}^{s} c_{i}^{\dagger} c_{j}
\end{aligned}
$$

$c_{i}^{\dagger}$ and $c_{i}$ are the creation and annihilation operators associated with the atomic spin orbital $\chi_{i}$. These orbitals are assumed to be orthonormal, ${ }^{7}$ i.e., $\left\langle\chi_{i} \mid \chi_{j}\right\rangle=\delta_{i j}$. These operators satisfy the Fermi anticommutation relationships

$$
\left[c_{i}^{\dagger}, c_{j}\right]_{+}=\delta_{i j} ; \quad\left[c_{i}^{\dagger}, c_{j}^{\dagger}\right]_{+}=0 ; \quad\left[c_{i}, c_{j}\right]_{+}=0 .
$$

Here the matrix $t$ represents the one-particle part of the electronic Hamiltonian, and the two-electron integrals are denoted by $\langle i j \mid k l\rangle \equiv(i k \mid j l)$. The singleelectron matrix elements $t_{i j}$, the two-electron matrix elements $(i j \mid k l)$, and the dipole moment matrix elements $\vec{\mu}_{i j}$ may be expressed in terms of the atomic

\footnotetext{
${ }^{7}$ Usually atomic orbitals basis sets are not orthonormal, i.e., $S_{i j} \equiv\left\langle\bar{\chi}_{i} \mid \bar{\chi}_{j}\right\rangle \neq \delta_{i j}$. The algebra is less involved if an orthonormal basis set is used, so that it is convenient to transform the nonorthonormal basis set $\left\{\bar{\chi}_{i}\right\}$ into an orthonormal one $\left\{\chi_{i}\right\}$, as follows:

$$
\begin{aligned}
\left|\chi_{i}\right\rangle & =\left|\bar{x}_{j}\right\rangle S_{j i}^{-1 / 2} ; \\
\left\langle\chi_{i}\right| & =S_{i j}^{-1 / 2}\left\langle\bar{x}_{j}\right| ; \\
\left\langle\chi_{i} \mid \chi_{j}\right\rangle & =S_{i k}^{-1 / 2}\left\langle\bar{x}_{k} \mid \bar{x}_{l}\right\rangle S_{l j}^{-1 / 2}=\left(S^{-1 / 2}\right)_{i k} S_{k l}\left(S^{-1 / 2}\right)_{l j}=\delta_{i j} .
\end{aligned}
$$
}


orbitals $\chi_{i}(\mathbf{r})$ as

$$
\begin{aligned}
t_{i j} & =\int \chi_{i}^{*}(\mathbf{r})\left[-\frac{1}{2} \nabla_{\mathbf{r}}^{2}+v(\mathbf{r})\right] \chi_{j}(\mathbf{r}) d \mathbf{r} ; \\
v(\mathbf{r}) & =-\sum_{I} \frac{Z_{I}}{\left|\mathbf{r}-\mathbf{R}_{I}\right|} ; \\
(i j \mid k l) & =\iint \frac{\chi_{i}^{*}\left(\mathbf{r}_{1}\right) \chi_{j}\left(\mathbf{r}_{1}\right) \chi_{k}^{*}\left(\mathbf{r}_{2}\right) \chi_{l}\left(\mathbf{r}_{2}\right)}{\left|\mathbf{r}_{1}-\mathbf{r}_{2}\right|} d \mathbf{r}_{1} d \mathbf{r}_{2} ; \\
\vec{\mu}_{i j} & =\int \chi_{i}^{*}(\mathbf{r}) \mathbf{r} \chi_{j}(\mathbf{r}) d \mathbf{r} .
\end{aligned}
$$

Invoking the Hartree-Fock approximation, the oneelectron ground state density matrix $(\bar{\rho})_{n m}=$ $\left\langle 0\left|c_{n}^{\dagger} c_{m}\right| 0\right\rangle$ satisfies the equation

$$
[F(\bar{q}), \bar{\rho}]=0,
$$

where the Fock operator $F$ is defined as follows:

$$
F(\rho)=t+V \rho .
$$

The action of the operator $V$ on the density matrix takes into account the interaction among electrons. For a closed shell system the action of $V$ on the Hartree-Fock self-consistent ground state density matrix $\bar{\rho}$ is (using the atomic orbital basis set):

$$
\begin{aligned}
(V \bar{\rho})_{i j} & =2(J \bar{\rho})_{i j}-(K \bar{\rho})_{i j} \\
(J \bar{\rho})_{i j} & =\sum_{k l}(i j \mid k l) \bar{\rho}_{k l} \\
(K \bar{\rho})_{i j} & =\sum_{k l}(i k \mid j l) \bar{\rho}_{k l} .
\end{aligned}
$$

$J$ and $K$ are commonly known as the Coulomb and the exchange operators, respectively.

The CEO equations may be derived by starting with the time-dependent Hartree-Fock equation

$$
\dot{\rho}(t)=-i[F(\rho), \rho] .
$$

The Fock matrix $F$ is time-dependent through $\rho(t)$. Assuming a small time-dependent deviation from the equilibrium ground state density matrix, $\rho(t)=$ $\bar{\rho}+\delta \rho(t)$, it is possible to derive directly from Eq. (47) the following linearized equation, where terms of order $\delta \rho^{2}$ have been neglected, and Eq. (42) has been considered:

$$
\dot{\delta} \rho=-i L \delta \rho .
$$

The operator $L \zeta=[F(\bar{\rho}), \zeta]+[V \zeta, \bar{\rho}]$ is defined over the space of single particle density matrices and represents an effective Liouville operator for the Hartree-Fock Hamiltonian. The Hermiticity of the density matrix $\delta \rho^{\dagger}=\delta \rho$ implies that its time derivative $\rho(t)=\dot{\delta} \rho(t)$ must be Hermitian at all times and that the Liouville operator must be anti-Hermitian

$$
(\dot{\delta} \rho)^{\dagger}=\dot{\delta} \rho \Rightarrow i L^{\dagger} \delta \rho=-i L \delta \rho \Rightarrow L^{\dagger}=-L .
$$

Let us consider now the spectrum of the $L$ operator (the frequencies $\Omega_{k}$ must be real to be physically meaningful in a system without dissipation)

$$
L \xi_{k}=\Omega_{k} \xi_{k} .
$$

Taking the Hermitian conjugate of this equation and making use of the anti-Hermiticity of $L$ we get

$$
L^{\dagger} \xi_{k}^{\dagger}=\Omega_{k} \xi_{k}^{\dagger} \Rightarrow L \xi_{k}^{\dagger}=-\Omega_{k} \xi_{k}^{\dagger} .
$$

The eigenvectors then come in conjugate pairs, $\xi_{k}$ and $\xi_{k}^{\dagger}$, with eigenvalues $\Omega_{k}$ and $-\Omega_{k}$, respectively. We introduce the following notation whereby positive and negative indexes refer to the positive and negative part of the spectrum $\left(\Omega_{k}\right.$ is taken to be positive when $k$ is positive):

$$
\xi_{k} \rightarrow \Omega_{k} \quad \xi_{-k} \equiv \xi_{k}^{\dagger} \rightarrow \Omega_{-k} \equiv-\Omega_{k} \quad \forall k>0 .
$$

The formal solution of Eq. (48) can be written as

$$
\delta \rho(t)=\exp (-i L t) \delta \rho(0) .
$$

The exponential of the Liouville operator works as a propagator of the initial perturbation on the ground state density matrix $\delta \rho(0)$. Its spectral representation is ( $\zeta$ represents a generic one particle matrix)

$$
\exp (-i L t) \zeta=\sum_{k>0 ; k<0} \xi_{k} e^{-i \Omega_{k} t}\left(\xi_{k}, \zeta\right)
$$

Acting with this propagator on $\delta \rho(0)$ we get

$$
\begin{aligned}
\delta \rho(t) & =\sum_{k>0 ; k<0} z_{k}(t) \xi_{k} ; \\
z_{k}(t) & =z_{k}(0) e^{-i \Omega_{k} t} ; \\
z_{k}(0) & =\left(\xi_{k}, \delta \rho(0)\right) .
\end{aligned}
$$

A scalar product $\left(\eta_{1}, \eta_{2}\right)$ of two one-electron density matrices in the Liouville space spanned by $L$ can be defined as

$$
\left(\eta_{1}, \eta_{2}\right) \equiv\left(\eta_{1} \mid \eta_{2}\right)=\operatorname{Tr}\left(\bar{\rho}\left[\eta_{1}^{\dagger}, \eta_{2}\right]\right)
$$

This is an unusual scalar product since it is antiHermitian, i.e., $\left(\eta_{1}, \eta_{2}\right)=-\left(\eta_{2}^{\dagger}, \eta_{1}^{\dagger}\right)$. With respect to this scalar product the Liouville operator $L$ becomes Hermitian: $\left(L \eta_{1}, \eta_{2}\right)=\left(\eta_{1}, L \eta_{2}\right)$. This can be explained as follows. Let us consider two vectors $\eta$ and $\zeta$ belonging to the Liouville space spanned by the $\mathrm{CEO}$ eigenvectors given by Eq. (50). We can write for the scalar product $(\eta, \zeta)$ the following expansion over the CEO eigenmodes:

$$
(\eta, \zeta)=\sum_{k}\left(\eta, \xi_{k}\right)\left(\xi_{k}, \zeta\right)
$$

Similarly the action of the Liouville operator $L$ on a generic vector $\varphi=\sum_{k} \xi_{k}\left(\xi_{k}, \varphi\right)$ can be repre- 
sented as

$$
L \varphi=\sum_{k} \Omega_{k} \xi_{k}\left(\xi_{k}, \varphi\right) .
$$

Making use of the above two expressions, and assuming $\zeta=L \varphi$, we can express $(\eta, L \varphi)$ as

$$
(\eta, L \varphi)=\sum_{k, h}\left(\eta, \xi_{k}\right)\left(\xi_{k}, \xi_{h}\right) \Omega_{h}\left(\xi_{h}, \varphi\right) .
$$

Using the anti-Hermicity of the scalar product $\left(\eta_{1}, \eta_{2}\right)=-\left(\eta_{2}^{\dagger}, \eta_{1}^{\dagger}\right)$, we obtain

$$
(\eta, L \varphi)=-\sum_{k, h}\left(\varphi^{\dagger}, \xi_{h}^{\dagger}\right)\left(\xi_{h}^{\dagger}, \xi_{k}^{\dagger}\right) \Omega_{k}\left(\xi_{k}^{\dagger}, \eta^{\dagger}\right) .
$$

Note that because of the orthogonality of the modes, $\left(\xi_{k}, \xi_{h}\right)=\delta_{k h}$, we are allowed to change $\Omega_{k}$ into $\Omega_{k}$ when going from Eq. (59) to Eq. (60). Making use of the notation with negative indexes, Eq. (52), we get

$$
(\eta, L \varphi)=\sum_{k, h}\left(\varphi^{\dagger}, \xi_{-h}\right)\left(\xi_{-h}, \xi_{-k}\right) \Omega_{-k}\left(\xi_{-k}, \eta^{\dagger}\right) .
$$

The sum over the CEO modes runs over both positive and negatives indexes; Eq. (61) is equivalent to

$$
(\eta, L \varphi)=\sum_{k, h}\left(\varphi^{\dagger}, \xi_{h}\right)\left(\xi_{h}, \xi_{k}\right) \Omega_{k}\left(\xi_{k}, \eta^{\dagger}\right) .
$$

The right-hand term of Eq. (62) is identified with $\left(\varphi^{\dagger}, L \eta^{\dagger}\right)$ through the use of Eq. (59), and we finally have

$$
(\eta, L \varphi)=\left(\varphi^{\dagger}, L \eta^{\dagger}\right)=-\left(L^{\dagger} \eta, \varphi\right)=(L \eta, \varphi) .
$$

The second equality holds because of the antiHermicity of the scalar product and the last one results from the anti-Hermicity of $L$.

The CEO algorithm is aimed at computing a few low-energy eigenvalues and the corresponding eigenvectors of the operator $L$ [Eq. (50)]. The computation is carried out with the Lanczos type algorithm discussed in [12]. According to Eq. (55) the CEO eigenvectors can be interpreted as electronic normal modes which represent the time evolution of the density matrix following an initial deviation from the equilibrium $\delta \rho(0)$. Another useful way to view the matrices $\xi_{k}$ comes from the expression of the linear response of the system in terms of $\xi_{k}$ [13]. By comparing the usual sum over states expression with the CEO expression involving the $\xi_{k}, \xi_{k}$ can be identified with the one-electron transition density matrix between the ground and the excited state of energy $\varepsilon_{k}=\Omega_{k}+\varepsilon_{0}$, i.e., $\left(\xi_{k}\right)_{n m}=\left\langle k\left|c_{n}^{\dagger} c_{m}\right| 0\right\rangle$. It is worth noting that if we express the density matrix as a single Slater determinant at all times (TDHF approximation), $\rho(t)$ becomes idempotent. Thus the matrix $\delta \rho(t)$ has to be a particle-hole matrix (the proof follows from Appendix E if a $\delta \rho=\bar{\rho}^{x} \delta x$ is considered). As a direct consequence the eigenvectors of the Liouville operator $L$ are also particle-hole matrices, see Eq. (55).

\section{Appendix B: TDHF Calculation of Nonadiabatic Couplings}

The NAC can be expressed in a form suitable for the TDHF applications making use of perturbation theory. According to the definition of the NAC, Eq. (2), we need to express the first derivative of the wavefunction with respect to the nuclear coordinate $x$. To that end we perturb the molecular Hamiltonian by introducing a small displacement $\Delta$ for a given nuclear coordinate $x$. To first order in $\Delta$ we have

$$
\hat{H}(x+\Delta)=\hat{H}(x)+\Delta \frac{\partial \hat{H}}{\partial x}+o\left(\Delta^{2}\right) .
$$

We can apply standard perturbation theory [22] to get the first-order approximations to the eigenvalues $\varepsilon_{k}(x+\Delta)$ and eigenvectors $|k(x+\Delta)\rangle$ of the perturbed (displaced) Hamiltonian $\hat{H}(x+\Delta)$. For the eigenvalues we have

$$
\varepsilon_{k}(x+\Delta)=\varepsilon_{k}(x)+\Delta\left\langle k(x)\left|\frac{\partial \hat{H}}{\partial x}\right| k(x)\right\rangle+o\left(\Delta^{2}\right) .
$$

This can be immediately used to calculate the derivative of the eigenvalue itself

$$
\begin{aligned}
\frac{\partial \varepsilon_{k}(x)}{\partial x} & =\lim _{\Delta \rightarrow 0} \frac{\varepsilon_{k}(x+\Delta)-\varepsilon_{k}(x)}{\Delta} \\
& =\left\langle k(x)\left|\frac{\partial \hat{H}}{\partial x}\right| k(x)\right\rangle .
\end{aligned}
$$

Equation (66) is the celebrated Hellmann-Feynman theorem for the computation of the atomic forces in molecules [23]. The same line of arguments can be used to calculate the derivative of the wavefunction as well. The first-order correction to the wavefunction is

$$
\begin{aligned}
|k(x+\Delta)\rangle & =|k(x)\rangle \\
& -\Delta \sum_{j>0 ; j \neq k}|j(x)| \frac{\left\langle j(x)\left|\frac{\partial \hat{H}}{\partial x}\right| k(x)\right|}{\varepsilon_{j}(x)-\varepsilon_{k}(x)}+o\left(\Delta^{2}\right) .
\end{aligned}
$$

The derivative is thus given by

$$
\begin{aligned}
\frac{\partial|k(x)\rangle}{\partial x} & =\lim _{\Delta \rightarrow 0} \frac{|k(x+\Delta)\rangle-|k(x)\rangle}{\Delta} \\
& =-\sum_{j>0 ; j \neq k}|j(x)| \frac{|j(x)| \frac{\partial \hat{H}}{\partial x}|k(x)\rangle}{\varepsilon_{j}(x)-\varepsilon_{k}(x)} .
\end{aligned}
$$


According to Eq. (2) we need to compute the derivative of the full many electron excited state wavefunction to get the NAG. We shall show that this can be done with a considerably reduced information (i.e., the one-particle transition density matrices obtained from a TDHF computation). Substituting the expression for the derivative of the wavefunction Eq. (68) into the definition of the NAG, Eq. (2), we get

$$
A_{i k}^{x}=\frac{\left\langle i\left|\hat{h}^{x}\right| k\right\rangle}{\varepsilon_{i}-\varepsilon_{k}} ; \quad i \neq k,
$$

where we have introduced the notation $\hat{h}^{x} \equiv \partial \hat{H} / \partial x$. It is important to note that this is a one-electron operator. Indeed, when the derivative of the molecular Hamiltonian is evaluated with respect to an arbitrary nuclear coordinate $x$, the two-electron part of the Hamiltonian involving the electron-electron repulsion does not depend on the position of the nuclei, and does not enter $\hat{h}^{x}$. When an atomic orbital basis set is employed, we have vibronic coupling also in the coefficients of the two-electron part of the Hamiltonian (39), i.e., $\langle i j \mid k l\rangle$ depends on the nuclear positions. This does not imply that $\hat{h}^{x}$ is a twoparticle operator, but the matrix elements of $\hat{h}^{x}$ in an atomic orbital representation will depend also on the derivative of the two-electrons integrals. $\hat{h}^{x}$ can thus be written in terms of the creation $\left(c_{n}^{\dagger}\right)$ and annihilation $\left(c_{m}\right)$ operators introduced earlier

$$
\frac{\partial \hat{H}}{\partial x} \equiv \hat{h}^{x}=\sum_{n m} h_{n m}^{x} c_{n}^{\dagger} c_{m} .
$$

To calculate the matrix elements of this operator entering Eq. (69) we can thus use the TDHF one-particle transition density matrices $\left(\rho_{i j}\right)_{n m}=$ $\left\langle i\left|c_{n}^{\dagger} c_{m}\right| j\right\rangle$, given in [13] in terms of the eigenvectors $\xi_{i}$ of the Liouville operator L. Introducing $\rho_{i j}$ and the transition energies $\Omega_{j}=\varepsilon_{j}-\varepsilon_{0}$ into Eq. (69) we get Eq. (4). The expression for NAC which involve the ground state, Eq. (5), is simpler and merely requires the knowledge of $\rho_{i 0} \equiv \xi_{i}$ and $\rho_{0 i} \equiv \xi_{i}^{\dagger}$, which are available from a CEO computation.

Thus TDHF computation of the NAC requires the explicit expression for the matrix elements of the one-electron operator $\hat{h}^{x}$, Eq. (70). This will be derived in Appendix C.

\section{Appendix C: The Expression for $\hat{h}^{x}$}

We can obtain a closed expression for the operator $\hat{h}^{x}$ in terms of the derivative of the matrix elements of the Hamiltonian with respect to an arbitrary nuclear displacement $x$. To that end we consider the perturbation on the Hartree-Fock solution for the ground state induced by a displacement along $x$. Applying the derivative operator $\partial / \partial x$ to the Hartree-Fock equation $[F(\bar{\rho}), \bar{\rho}]=0$ we get (see Appendix $\mathrm{H}$ for the explicit definition of the derivatives of the various terms)

$$
\begin{aligned}
\frac{\partial}{\partial x}[t+V \bar{\rho}, \bar{\rho}] & =\left[t^{x}+V^{x} \bar{\rho}+V \bar{\rho}^{x}, \bar{\rho}\right]+\left[t+V \bar{\rho}, \bar{\rho}^{x}\right] \\
& =\left[F(\bar{\rho}), \bar{\rho}^{x}\right]+\left[V \bar{\rho}^{x}, \bar{\rho}\right]+\left[t^{x}+V^{x} \bar{\rho}, \bar{\rho}\right] \\
& =L \bar{\rho}^{x}-\left[\bar{\rho}, t^{x}+V^{x} \bar{\rho}\right]=0 .
\end{aligned}
$$

The same Liouville operator $L$ that describes electronic excitations induced by an external electric field $[6,15]$, also represents the perturbation on the density matrix induced by a small displacement of nuclear coordinates:

$$
L \bar{\rho}^{x}=\left[\bar{\rho}, t^{x}+V^{x} \bar{\rho}\right] .
$$

The effect of this displacement is equivalent to adding an external field $\Delta$ that perturbs the isolated molecule Hamiltonian (64) through the operator $\hat{h}^{x}$ instead of the dipole operator $\hat{\mu}$ coupled to the electric field $\mathcal{E}$. This allows us to identify the matrix elements of $\hat{h}^{x}$ with $t^{x}+V^{x} \bar{\rho} .^{8}$ This can be put on a firm basis as shown below. The derivative of the idempotency condition of $\bar{\rho}$ implies that $\bar{\rho}^{x}$ belongs to the particle-hole space (see Appendix E), so that it may be expanded in terms of the eigenvectors of $L$

$$
\bar{\rho}^{x}=\sum_{j>0} a_{j}^{x} \xi_{j}+b_{j}^{x} \xi_{j}^{\dagger} .
$$

These eigenvectors are orthonormal and they come in conjugate pairs $\xi_{j}, \xi_{j}^{\dagger}$ with eigenvalues $\Omega_{j}$ and $-\Omega_{j}$, respectively. Using the scalar product that makes the Liouville operator Hermitian, Eq. (56), we have the following orthonormality relations for a given $\mathrm{CEO}$ eigenvector:

$$
\begin{aligned}
(\xi, \xi) & =\operatorname{Tr}\left(\bar{\rho}\left[\xi^{\dagger}, \xi\right]\right)=1 ; \\
\left(\xi^{\dagger}, \xi^{\dagger}\right) & =\operatorname{Tr}\left(\bar{\rho}\left[\xi, \xi^{\dagger}\right]\right)=-1 ; \\
\left(\xi, \xi^{\dagger}\right) & =\operatorname{Tr}\left(\bar{\rho}\left[\xi^{\dagger}, \xi^{\dagger}\right]\right)=0 ; \\
\left(\xi^{\dagger}, \xi\right) & =\operatorname{Tr}(\bar{\rho}[\xi, \xi])=0 .
\end{aligned}
$$

To compute the coefficients $a_{j}^{x}$ and $b_{j}^{x}$ of Eq. (73), we consider the scalar product of Eq. (72) with the

\footnotetext{
${ }^{8}$ The equation for the first-order perturbation $\xi(t)$ to the ground state density matrix $\bar{\rho}$ with respect to an electric field $\mathcal{E}(t)$ is: $i[\partial \xi(t) / \partial t]-L \xi(t)=-\mathcal{E}(t)[\boldsymbol{\mu}, \bar{\rho}]$ [6]. In the limit of a static field $\mathcal{E}$ this reduces to $L \xi=\mathcal{E}[\bar{\rho}, \mu]$.
} 
eigenvectors $\xi_{j}$ and $\xi_{j}^{\dagger}$

$$
\begin{aligned}
\left(\xi_{j}, L \bar{\rho}^{x}\right) & =\left(\xi_{j},\left[\bar{\rho}, t^{x}+V^{x} \bar{\rho}\right]\right) ; \\
\left(\xi_{j}^{\dagger}, L \bar{\rho}^{x}\right) & =\left(\xi_{j}^{\dagger},\left[\bar{\rho}, t^{x}+V^{x} \bar{\rho}\right]\right) .
\end{aligned}
$$

Substituting the expansion of $\bar{\rho}^{x}$ into Eqs. (75), making use of the orthonormality conditions (74) for the left-hand side of Eqs. (75) we get

$$
\begin{aligned}
\left(\xi_{j}, L \bar{\rho}^{x}\right) & =\left(\xi_{i}, L \sum_{i>0} a_{i}^{x} \xi_{i}+b_{i}^{x} \xi_{i}^{\dagger}\right) \\
& =\left(\xi_{j}, \sum_{i>0} a_{i}^{x} \Omega_{i} \xi_{i}-b_{i}^{x} \Omega_{i} \xi_{i}^{\dagger}\right) \\
& =a_{j}^{x} \Omega_{j}\left(\xi_{j}, \xi_{j}\right)=\Omega_{j} a_{j}^{x},
\end{aligned}
$$

and

$$
\begin{aligned}
\left(\xi_{j}^{\dagger}, L \bar{\rho}^{x}\right) & =\left(\xi_{i}^{\dagger}, L \sum_{i>0} a_{i}^{x} \xi_{i}+b_{i}^{x} \xi_{i}^{\dagger}\right) \\
& =\left(\xi_{j}^{\dagger}, \sum_{i>0} a_{i}^{x} \Omega_{i} \xi_{i}-b_{i}^{x} \Omega_{i} \xi_{i}^{\dagger}\right) \\
& =-b_{j}^{x} \Omega_{j}\left(\xi_{j}^{\dagger}, \xi_{j}^{\dagger}\right)=\Omega_{j} b_{j}^{x} .
\end{aligned}
$$

This yields the following expression for the coefficients:

$$
\begin{aligned}
& a_{j}^{x}=\frac{\left(\xi_{j},\left[\bar{\rho}, t^{x}+V^{x} \bar{\rho}\right]\right)}{\Omega_{j}} ; \\
& b_{j}^{x}=\frac{\left(\xi_{j}^{\dagger},\left[\bar{\rho}, t^{x}+V^{x} \bar{\rho}\right]\right)}{\Omega_{j}} .
\end{aligned}
$$

Since both $\bar{\rho}$ and $t^{x}+V^{x} \bar{\rho}$ are Hermitian, Eq. (78) shows that the coefficients, like the eigenmodes, come in conjugate pairs, $b_{j}^{x}=\left(a_{j}^{x}\right)^{\dagger}$. This guarantees that the derivative of the density matrix is also Hermitian. As must be the case because $\bar{\rho}^{x}$ is the derivative of the Hermitian matrix $\bar{\rho}$. Perturbative calculation gives for the derivative of the density matrix (see Appendix D)

$$
\bar{\rho}^{x}=-\sum_{j>0} \frac{\operatorname{Tr}\left(\xi_{j} h^{x}\right) \xi_{j}^{\dagger}+\operatorname{Tr}\left(\xi_{j}^{\dagger} h^{x}\right) \xi_{j}}{\Omega_{j}} .
$$

$\bar{\rho}^{x}$ is thus expanded over the basis set of the CEO transition density matrices $\xi_{j}, \xi_{j}^{\dagger}$. Comparing the result of the expansion carried out using the coefficients $a_{j}^{x}$ and $b_{j}^{x}$. [Eq. (78)] with Eq. (79) we can make the following identifications:

$$
\begin{aligned}
& a_{j}^{x} \rightarrow\left(\xi_{j},\left[\bar{\rho}, t^{x}+V^{x} \bar{\rho}\right]\right)=-\operatorname{Tr}\left(\xi_{j}^{\dagger} h^{x}\right) ; \\
& b_{j}^{x} \rightarrow\left(\xi_{j}^{\dagger},\left[\bar{\rho}, t^{x}+V^{x} \bar{\rho}\right]\right)=-\operatorname{Tr}\left(\xi_{j} h^{x}\right) .
\end{aligned}
$$

Finally, using the definition of the scalar product $\left(\zeta_{1}, \zeta_{2}\right)$ and expanding the left-hand term of the above equalities, we obtain Eq. (6) (see Appendix F).

\section{Appendix D: Derivative of the Single-Electron Ground State Density Matrix}

Using the perturbative expression for the derivative of the wavefunction, we can immediately get a closed expression for the derivative of the singleelectron density matrix. Considering the wavefunction approximated by a single Slater determinant, we can apply the standard definition of the oneelectron density matrix in the second quantized form: $(\bar{\rho})_{n m}=\left\langle 0\left|c_{n}^{\dagger} c_{m}\right| 0\right\rangle$. The derivative of the density matrix can be carried out

$$
\begin{aligned}
\left(\bar{\rho}^{x}\right)_{n m} & =\frac{\partial}{\partial x}\left\langle 0\left|c_{n}^{\dagger} c_{m}\right| 0\right\rangle \\
& =\frac{\partial\langle 0|}{\partial x} c_{n}^{\dagger} c_{m}|0\rangle+\langle 0| c_{n}^{\dagger} c_{m} \frac{\partial|0\rangle}{\partial x} .
\end{aligned}
$$

To evaluate this expression we need the derivative of the ground state bra and ket. Making use of Eq. (68), we have

$$
\begin{aligned}
& \frac{\partial|0\rangle}{\partial x}=-\sum_{j>0}|j\rangle \frac{\left\langle j\left|\hat{h}^{x}\right| 0\right\rangle}{\Omega_{j}} ; \\
& \frac{\partial\langle 0|}{\partial x}=-\sum_{j>0} \frac{\left\langle 0\left|\hat{h}^{x}\right| j\right\rangle}{\Omega_{j}}\langle j| .
\end{aligned}
$$

We have denoted the transition energy from the ground state as $\Omega_{j}=\varepsilon_{j}-\varepsilon_{0}$. Substituting Eq. (82) into Eq. (81) we obtain for the derivative of the ground state density matrix

$$
\left(\bar{\rho}^{x}\right)_{n m}=-\sum_{j>0} \frac{\left\langle 0\left|\hat{h}^{x}\right| j\right\rangle}{\Omega_{j}}\left\langle j\left|c_{n}^{\dagger} c_{m}\right| 0\right\rangle+\left\langle 0\left|c_{n}^{\dagger} c_{m}\right| j\right\rangle \frac{\left\langle j\left|\hat{h}^{x}\right| 0\right\rangle}{\Omega_{j}} .
$$

Finally adopting the definition of the CEO eigenmodes $\left(\xi_{j}\right)_{n m}=\left\langle j\left|c_{n}^{\dagger} c_{m}\right| 0\right\rangle,\left(\xi_{j}\right)_{n m}^{\dagger}=\left\langle 0\left|c_{n}^{\dagger} c_{m}\right| j\right\rangle$, and the second quantized version of $\hat{h}^{x}=\sum_{n m} h_{n m}^{x} c_{n}^{\dagger} c_{m}$, we get

$$
\bar{\rho}^{x}=-\sum_{j>0} \frac{\operatorname{Tr}\left(\xi_{j}^{\dagger} h^{x}\right) \xi_{j}+\operatorname{Tr}\left(\xi_{j} h^{x}\right) \xi_{j}^{\dagger}}{\Omega_{j}} .
$$

Note that the derivative of the density matrix turns out to be Hermitian: $\left(\bar{\rho}^{x}\right)^{\dagger}=\bar{\rho}^{x}$ (the operator $\hat{h}^{x}$ is Hermitian). This is the direct consequence of the Hermiticity of the density matrix. Using the same approach it is possible to derive closed expressions for the derivative of excited states density matrices and transition density matrices over excited states. 
The final result is

$$
\left(\rho_{i j}^{x}\right)=\sum_{k \neq i} \frac{\left\langle i\left|\hat{h}^{x}\right| k\right\rangle}{\varepsilon_{i}-\varepsilon_{k}}\left(\rho_{k j}\right)_{n m}-\sum_{k \neq j}\left(\rho_{i k}\right)_{n m} \frac{\left\langle k\left|\hat{h}^{x}\right| j\right\rangle}{\varepsilon_{k}-\varepsilon_{j}} .
$$

By substituting the NAG from Eq. (69) in the above expression for $\rho_{i j}^{x}$, we obtain Eq. (7). This shows that it is possible to expand the derivative of the general transition density matrix in terms of transition density matrices (vectors in Liouville space) where the coefficients are given by the NAG.

\section{Appendix E: Representation of $\bar{\rho}^{x}$ in the CEO Basis Set}

The derivative of the idempotency condition $\bar{\rho}^{2}=\bar{\rho}$ implies that the derivative of the density matrix $\bar{\rho}^{x}$ belongs to the space spanned by the CEO eigenvectors (the particle-hole subspace of the single Slater determinant space). To show that we start with the derivative of the idempotency

$$
\bar{\rho}^{x} \bar{\rho}+\bar{\rho} \bar{\rho}^{x}=\bar{\rho}^{x} .
$$

We adopt the molecular orbital representation for the density matrices ${ }^{9}$

$$
\bar{\rho}=\left(\begin{array}{cc}
0 & 0 \\
0 & 1
\end{array}\right) ; \quad \bar{\rho}^{x}=\left(\begin{array}{ll}
a & b \\
c & d
\end{array}\right) .
$$

Expanding the matrix products in Eq. (86) using this representation we get

$$
\left(\begin{array}{cc}
0 & b \\
c & 2 d
\end{array}\right)=\left(\begin{array}{ll}
a & b \\
c & d
\end{array}\right)
$$

This immediately implies that the blocks $a$ and $d$ must be zero. The structure of $\bar{\rho}^{x}$ is then block-off diagonal, the same as the CEO eigenvectors.

\section{Appendix F: Identification of $h^{x}$}

In order to identify the matrix elements of $h^{x}$ we have to consider Eqs. (80) and expand the scalar product on the left-hand side. Below we show that this scalar product $\left(\xi_{j},\left[\bar{\rho}, t^{x}+V^{x} \bar{\rho}\right]\right)$ is given by $-\operatorname{Tr}\left\{\xi_{j}^{\dagger}\left(t^{x}+V^{x} \bar{\rho}\right)\right\}$, so that Eqs. (80) reduce to

$$
\begin{aligned}
a_{j}^{x} \rightarrow\left(\xi_{j},\left[\bar{\rho}, t^{x}+V^{x} \bar{\rho}\right]\right) & =-\operatorname{Tr}\left\{\xi_{j}^{\dagger}\left(t^{x}+V^{x} \bar{\rho}\right)\right\} \\
& =-\operatorname{Tr}\left(\xi_{j}^{\dagger} h^{x}\right) .
\end{aligned}
$$

\footnotetext{
${ }^{9}$ In this representation the blocks are identified as follows: $\left(\begin{array}{l}\text { UU UO } \\ \text { OU OO }\end{array}\right)$. Occupied MO's are labeled $O$ and unoccupied MO's are labeled $U$.
}

The equation for $b_{j}^{x}$ is redundant and it is simply the conjugate of the equation for $a_{j}^{x}$. Equation (89) finally gives the expression for $h^{x}=t^{x}+V^{x} \bar{\rho}$.

Let us represent our matrices in the molecular orbital basis set

$$
\begin{array}{ll}
\xi_{j}=\left(\begin{array}{cc}
0 & \alpha \\
\beta & 0
\end{array}\right) ; \quad \xi_{j}^{\dagger}=\left(\begin{array}{cc}
0 & \beta^{\dagger} \\
\alpha^{\dagger} & 0
\end{array}\right) ; \\
\bar{\rho}=\left(\begin{array}{cc}
0 & 0 \\
0 & 1
\end{array}\right) & t^{x}+V^{x} \bar{\rho}=\left(\begin{array}{cc}
A & B \\
C & D
\end{array}\right) .
\end{array}
$$

Expanding the scalar product in Eqs. (80) using its definition, Eq. (56), we have to compute the trace of a nested pair of commutators multiplied by the density matrix

$$
\left(\xi_{j},\left[\bar{\rho}, t^{x}+V^{x} \bar{\rho}\right]\right) \equiv \operatorname{Tr}\left(\bar{\rho}\left[\xi_{j}^{\dagger},\left[\bar{\rho}, t^{x}+V^{x} \bar{\rho}\right]\right]\right) .
$$

We shall compute the right-hand side of Eq. (91) step by step. The inner commutator can be expanded as follows:

$$
\left[\bar{\rho}, t^{x}+V^{x} \bar{\rho}\right]=\left(\begin{array}{cc}
0 & -B \\
C & 0
\end{array}\right) .
$$

Making use of the above expression, the outer commutator gives

$$
\left[\xi_{j}^{\dagger},\left(\begin{array}{cc}
0 & -B \\
C & 0
\end{array}\right)\right]=\left(\begin{array}{cc}
\beta^{\dagger} C+B \alpha^{\dagger} & 0 \\
0 & -\alpha^{\dagger} B-C \beta^{\dagger}
\end{array}\right) .
$$

Multiplying by $\bar{\rho}$ and taking the trace we finally obtain

$$
\begin{aligned}
& \operatorname{Tr}\left(\bar{\rho}\left[\xi_{j}^{\dagger},\left[\bar{\rho}, t^{x}+V^{x} \bar{\rho}\right]\right]\right) \\
& =\operatorname{Tr}\left\{\left(\begin{array}{ll}
0 & 0 \\
0 & 1
\end{array}\right)\left(\begin{array}{cc}
\beta^{\dagger} C+B \alpha^{\dagger} & 0 \\
0 & -\alpha^{\dagger} B-C \beta^{\dagger}
\end{array}\right)\right\} \\
& \quad-\operatorname{Tr}\left(\alpha^{\dagger} B\right)-\operatorname{Tr}\left(C \beta^{\dagger}\right) .
\end{aligned}
$$

It is easy to show that except for a minus sign the same result can be obtained from the following simpler formula:

$$
\begin{aligned}
\operatorname{Tr}\left\{\xi_{j}^{\dagger}\left(t^{x}+V^{x} \bar{\rho}\right)\right\} & =\operatorname{Tr}\left\{\left(\begin{array}{cc}
0 & \beta^{\dagger} \\
\alpha^{\dagger} & 0
\end{array}\right)\left(\begin{array}{cc}
A & B \\
C & D
\end{array}\right)\right\} \\
& =\operatorname{Tr}\left(\begin{array}{cc}
\beta^{\dagger} C & \beta^{\dagger} D \\
\alpha^{\dagger} A & \alpha^{\dagger} B
\end{array}\right) \\
& =\operatorname{Tr}\left(C \beta^{\dagger}\right)+\operatorname{Tr}\left(\alpha^{\dagger} B\right) .
\end{aligned}
$$

We have made use of the cyclic invariance of the trace, $\operatorname{Tr}(A B)=\operatorname{Tr}(B A)$. This proves the first equality in Eq. (89). 


\section{Appendix G: Derivative of a General Particle-Hole Matrix}

To discuss the variation $\delta \xi$ of a general interband matrix $\xi$ induced by a change $\delta \bar{\rho}$ of the ground state density matrix ${ }^{10}$ it is convenient to introduce a projection operator $P$ in the Liouville space spanned by the $\mathrm{CEO}$ eigenvectors. $P$ projects a general matrix into the particle-hole subspace (i.e., the off diagonal block involving the occupied-unoccupied molecular orbitals). $P$ is given by

$$
P \zeta \equiv[[\zeta, \bar{\rho}], \bar{\rho}] .
$$

When $P$ acts on an arbitrary matrix in the single electron space $\zeta=\left(\begin{array}{c}\zeta_{\text {UU }} \zeta_{\text {UO }} \\ \zeta_{\mathrm{OU}} \zeta_{\mathrm{OO}}\end{array}\right)$ it extracts the particle-hole component

$$
P \zeta=\left(\begin{array}{cc}
0 & \zeta \text { UO } \\
\zeta \mathrm{OU} & 0
\end{array}\right)
$$

As must be the case for any projection operator, $P$ is idempotent: $P^{2}=P$. For a general interband matrix $\xi$ the following condition must be true:

$$
P \xi=\xi, \quad \text { i.e., }[[\xi, \bar{\rho}], \bar{\rho}]=\xi \text {. }
$$

A change $\delta \bar{\rho}$ in the density matrix $\bar{\rho}$, is accompanied by a change in the associated particle-hole space. Let us consider the following displacements for the density matrix and for the general $\xi$ matrix in the occupied-unoccupied molecular orbital representation:

$$
\begin{aligned}
\bar{\rho}+\delta \bar{\rho}= & \left(\begin{array}{ll}
0 & 0 \\
0 & 1
\end{array}\right)+\left(\begin{array}{cc}
0 & \delta \bar{\rho} \\
\delta \bar{\rho}^{\dagger} & 0
\end{array}\right) \\
\xi+\delta \xi= & \xi+(\delta \gamma+\delta \varphi)=\left(\begin{array}{cc}
0 & \beta \\
\alpha & 0
\end{array}\right) \\
& +\left(\begin{array}{cc}
0 & \delta \beta \\
\delta \alpha & 0
\end{array}\right)+\left(\begin{array}{cc}
\delta \varphi_{2} & 0 \\
0 & \delta \varphi_{1}
\end{array}\right) .
\end{aligned}
$$

The variation of the interband matrix $\xi$ consists of the particle-hole component $\delta \gamma$ and the component outside the particle-hole space $\delta \varphi$. It is possible to express $\delta \varphi$ in terms of $\delta \bar{\rho}$. To that end suffice it to write Eq. (99) using the displaced quantities

$$
[[\xi+\delta \xi, \bar{\rho}+\delta \bar{\rho}], \bar{\rho}+\delta \bar{\rho}]=\xi+\delta \xi .
$$

\footnotetext{
${ }^{10}$ A nuclear displacement $\delta x$ can be considered to be the cause of the variation $\delta \bar{\rho}$.
}

Let us compute the inner commutator of Eq. (102) at first order in $\delta \bar{\rho}$ and $\delta \xi$

$$
\begin{aligned}
K+\delta K \approx[\xi & +\delta \xi, \bar{\rho}+\delta \bar{\rho}] \approx\left(\begin{array}{cc}
0 & \beta \\
-\alpha & 0
\end{array}\right) \\
& +\left(\begin{array}{cc}
\beta \delta \bar{\rho}^{\dagger}-\delta \bar{\rho} \alpha & \delta \beta \\
-\delta \alpha & \alpha \delta \bar{\rho}-\delta \bar{\rho}^{\dagger} \beta
\end{array}\right) .
\end{aligned}
$$

Making use of Eq. (103) it is easy to compute the two terms of the double commutator (102). For the particle-hole component this merely results in an identity with respect to Eq. (101):

$$
[K+\delta K, \bar{\rho}]=\left(\begin{array}{cc}
0 & \beta+\delta \beta \\
\alpha+\delta \alpha & 0
\end{array}\right)=\xi+\delta \gamma .
$$

The useful information comes from the particleparticle/hole-hole component

$$
\begin{aligned}
& {[K+\delta K, \delta \bar{\rho}] \approx[K, \delta \bar{\rho}]} \\
& \quad=\left(\begin{array}{cc}
\beta \delta \bar{\rho}^{\dagger}+\delta \bar{\rho} \alpha & 0 \\
0 & -(\delta \bar{\rho} \beta+\alpha \delta \bar{\rho})
\end{array}\right)=\delta \varphi .
\end{aligned}
$$

To cast Eq. (105) in a standard matrix form, we make use of the following simple relationships:

$$
\xi \delta \bar{\rho}=\left(\begin{array}{cc}
\beta \delta \bar{\rho}^{\dagger} & 0 \\
0 & \alpha \delta \bar{\rho}
\end{array}\right) ; \quad \delta \bar{\rho} \xi=\left(\begin{array}{cc}
\delta \bar{\rho} \alpha & 0 \\
0 & \delta \bar{\rho}^{\dagger} \beta
\end{array}\right) .
$$

We further recall how the density matrix itself acts on the particle-particle/hole-hole space

$$
\begin{aligned}
\bar{\rho}\left(\begin{array}{cc}
U U & 0 \\
0 & O O
\end{array}\right) & =\left(\begin{array}{cc}
0 & 0 \\
0 & 0 O
\end{array}\right) ; \\
(1-\bar{\rho})\left(\begin{array}{cc}
U U & 0 \\
0 & O O
\end{array}\right) & =\left(\begin{array}{cc}
U U & 0 \\
0 & 0
\end{array}\right) .
\end{aligned}
$$

Making use of Eqs. (106), (107) into Eq. (105) we get the final expression for the particle-particle/ hole-hole component of the variation of a general interband matrix $\xi$

$$
\delta \varphi=(1-2 \bar{\rho})(\xi \delta \bar{\rho}+\delta \bar{\rho} \xi)=(1-2 \bar{\rho})[\xi, \delta \bar{\rho}]_{+} .
$$

Equation (108) can be used to express the intraband component $\varphi_{i}^{x}$ of the derivative of a CEO eigenvector $\xi_{i}^{x}$, e.g., $\varphi_{i}^{x}=\delta \varphi_{i} / \delta x$. This result is used in Eq. (20) of the third section.

Let us consider now the equation for the derivative of the CEO eigenvector, Eq. (18). In order to solve this equation for $\xi_{i}^{x}$, we need to consider the projection operator $P$ already defined with Eq. (97). Acting with $P$ on $\xi_{i}^{x}$ projects out the particle-hole component: $P \xi_{i}^{x}=\gamma_{i}^{x}$. It is worth noting that $P$ and $L$ share the same eigenvectors so that they commute. ${ }^{11}$

\footnotetext{
${ }^{11}$ Any eigenvector $\xi$ of $L$ is a particle-hole matrix, so that $P \xi=\xi[15]$.
} 
The shifted Liouville operator $L-\Omega_{i}$ has the entire spectrum of eigenvalues shifted by $-\Omega_{i}$, but the eigenvectors are still the same as those of $L$. Thus also $L-\Omega_{i}$ commutes with $P$. Keeping this in mind we can now directly apply $P$ to Eq. (18). The action of $P$ on the left-hand side gives

$$
P\left\{\left(L-\Omega_{i}\right) \xi_{i}^{x}\right\}=\left(L-\Omega_{i}\right) P\left(\gamma_{i}^{x}+\varphi_{i}^{x}\right)=\left(L-\Omega_{i}\right) \gamma_{i}^{x} .
$$

The action of $P$ on the right-hand side gives

$$
P C_{i}^{x} \equiv D_{i}^{x}=\left[\left[C_{i}^{x}, \bar{\rho}\right], \bar{\rho}\right] .
$$

Thus the projection of Eq. (18) in the particle-hole space is

$$
\left(L-\Omega_{i}\right) \gamma_{i}^{x}=D_{i}^{x} .
$$

This projection can be now expressed in terms of the eigenmodes $\xi_{i}$ of the Liouville operator. Solving Eq. (111) requires inverting the Liouville operator $L$. If this operator is represented in terms of its spectrum, $L \zeta=\sum_{k>0 ; k<0} \xi_{k} \Omega_{k}\left(\xi_{k}, \zeta\right)$, the inversion becomes straightforward. The shifted Liouville operator in Eq. (18) can be written as

$$
\left(L-\Omega_{i}\right) \zeta=\sum_{k>0 ; k<0} \xi_{k}\left[\Omega_{k}-\Omega_{i}\right]\left(\xi_{k}, \zeta\right)
$$

and its inverse is

$$
\left(L-\Omega_{i}\right)^{-1} \zeta=\sum_{k>0 ; k<0} \frac{\xi_{k}\left(\xi_{k}, \zeta\right)}{\Omega_{k}-\Omega_{i}}
$$

where the prime denotes that the $i$ th mode is excluded from the sum. This eliminates divergences and is consistent with the fact that the derivative $\xi_{i}^{x}$, and so $\gamma_{i}^{x}$, has no projection on $\xi_{i}$ [3]. This definition of the inverse in a space orthogonal to the subspace spanned by the vector $\xi_{i}$ alone can thus be safely used to compute $\gamma_{i}^{x}$. This is obtained directly by using this inverse, so that $\gamma_{i}^{x}=\left(L-\Omega_{i}\right)^{-1} D_{i}^{x}$. The result is the following final expression:

$$
\gamma_{i}^{x}=\sum_{k>0 ; k<0} \frac{\xi_{k}\left(\xi_{k}, D_{i}^{x}\right)}{\Omega_{k}-\Omega_{i}} .
$$

Eq. (114) is used in Eq. (20) of the third section to compute the $\mathrm{CEO}$ eigenvector derivative, $\xi_{i}^{x}$.

\section{Appendix H: Derivative of the Fock Matrix}

Computing the derivative of the Fock matrix $F$ with respect to a nuclear coordinate $x$ is required for deriving the closed expression for the operator $\hat{h}^{x}$ (see Appendix C). This computation involves the derivative of the one-electron part and the electron repulsion term of the Hamiltonian. Applying the derivative $\partial / \partial x$ on the matrix elements of $F_{i j}=$ $t_{i j}+(V \rho)_{i j}$ we have

$$
F_{i j}^{x}=t_{i j}^{x}+\left(V^{x} \rho\right)_{i j}+\left(V \rho^{x}\right)_{i j} .
$$

We consider a generic density matrix, not necessarily the self-consistent Hartree-Fock one, $\bar{\rho}$. For the one-electron matrix elements we have

$$
\left(t^{x}\right)_{i j}=\frac{\partial t_{i j}}{\partial x} .
$$

The derivative of the two-electron term is given by

$$
\begin{aligned}
\left(V^{x} \rho\right)_{i j} & =2\left(J^{x} \rho\right)_{i j}-\left(K^{x} \rho\right)_{i j} \\
\left(J^{x} \rho\right)_{i j} & =\sum_{k l}(i j \mid k l)^{x} \rho_{k l} \\
\left(K^{x} \rho\right)_{i j} & =\sum_{k l}(i k \mid j l)^{x} \rho_{k l}
\end{aligned}
$$

and

$$
\begin{aligned}
\left(V \rho^{x}\right)_{i j} & =2\left(J \rho^{x}\right)_{i j}-\left(K \rho^{x}\right)_{i j} \\
\left(J \rho^{x}\right)_{i j} & =\sum_{k l}(i j \mid k l) \rho_{k l}^{x} \\
\left(K \rho^{x}\right)_{i j} & =\sum_{k l}(i k \mid j l) \rho_{k l}^{x} .
\end{aligned}
$$

We have defined the derivative of the two-electron integral

$$
(i j \mid k l)^{x} \equiv \frac{\partial(i j \mid k l)}{\partial x} .
$$

For a basis set independent on the nuclear coordinates (e.g., plane waves commonly used in density functional calculations), the only source of vibronic coupling comes from the one-electron matrix elements $t_{i j}$ through the potential integral $\int \chi_{i}^{*}(\mathbf{r}) v(\mathbf{r}) \chi_{j}(\mathbf{r}) d \mathbf{r}$. In standard quantum chemistry computations a geometry-dependent atomic orbital basis set is commonly used, so that the vibronic couplings enter also the two-electron matrix elements $(i j \mid k l)$. This is the reason why the one-electron operator $\hat{h}^{x}$ described in the fifth section contains $V^{x}$ as well.

Finally we note that the derivatives $t^{x}$ and $(i j \mid k l)^{x}$ introduced insofar are expressed in the orthonormal atomic orbital basis set. When a nonorthonormal basis is used, the relationships between the atomic matrix elements in the two basis sets can be directly 
used (see Note 7)

$$
\begin{aligned}
t_{i j} & =\left\langle\chi_{i}|\hat{t}| \chi_{j}\right\rangle=S_{i k}^{-1 / 2}\left\langle\tilde{\chi}_{k}|\hat{t}| \tilde{\chi}_{l}\right\rangle S_{l j}^{-1 / 2} \\
& =S_{i k}^{-1 / 2} \tilde{t}_{k l} S_{l j}^{-1 / 2} ; \\
\langle i j \mid k l\rangle & =S_{i i^{\prime}}^{-1 / 2} S_{j j^{\prime}}^{-1 / 2}\left\langle i^{\prime} j^{\prime} \mid k^{\prime} l^{\prime}\right\rangle S_{k^{\prime} k}^{-1 / 2} S_{l^{\prime} l}^{-1 / 2} .
\end{aligned}
$$

A relationship between the derivatives of the matrix elements in the two basis sets can be obtained by differentiating these equations. $t_{i j}^{x}$ and $\langle i j \mid k l\rangle^{x}$ will involve the derivative of the overlap matrix $S$; for example for the one-electron matrix elements we have

$$
t^{x}=\left(S^{-1 / 2}\right)^{x} \tilde{t} S^{-1 / 2}+S^{-1 / 2} \tilde{t}^{x} S^{-1 / 2}+S^{-1 / 2} \tilde{t}\left(S^{-1 / 2}\right)^{x} .
$$

\section{ACKNOWLEDGMENTS}

The support of the National Science Foundation and the Petroleum Research Fund sponsored by the American Chemical Society are gratefully acknowledged. We wish to thank Dr. M. Ottonelli for many fruitful discussions.

\section{References}

1. Domcke, W.; Stock, G. Adv Chem Phys 1991, 100, 1-169.

2. Lengsfield III, B. H.; Yarkony, D. R. Adv Chem Phys 1992, $82,1-71$.

3. Tsiper, E. V.; Chernyak, V.; Tretiak, S.; Mukamel, S. J Chem Phys 1999, 110, 8328-8337.

4. Tommasini, M.; Chernyak, V.; Mukamel, S. In Ultrafast Phenomena XII; Elsasser, T.; Mukamel, S.; Murnane, M.; Scherer, N., Eds.; Springer-Verlag: Berlin, 2000.
5. Deumens, E.; Diz, A.; Longo, R.; Ohrn, Y. Rev Mod Phys 1994, 66, 917.

6. Tretiak, S.; Chernyak, V.; Mukamel, S. J Am Chem Soc 1997, 119, 11408-11419.

7. Ben-Num, M.; Martinez, T. J. J Chem Phys 1998, 108, 7244; Ben-Num, M.; Martinez, T. J. Chem Phys 2000, 259, 237.

8. Wald, G. Science 1968, 162, 230.

9. Schulten, K.; Humphrey, W.; Logunov, I.; Sheves, M.; Xu, D. Isr J Chem 1995, 35, 447.

10. Molnar, F.; Ben-Nun, M.; Martinez, T. J.; et al. J Mol Struct (THEOCHEM) 2000, 506, 169; Ben-Nun, M.; Molnar, F.; Lu, H.; et al. Faraday Discuss 1998, 110, 447.

11. Chernyak, V.; Mukamel, S. J Chem Phys 2000, 112, 35723579.

12. Chernyak, V.; Schulz, M. F.; Mukamel, S.; Tretiak, S.; Tsiper, E. V. J Chem Phys 2000, 113, 36-43.

13. Tretiak, S.; Chernyak, V.; Mukamel, S. Int J Quantum Chem 1998, 70, 711-727.

14. Car, R.; Parrinello, M. Phys Rev Lett 1985, 55, 2471; for a review see Remler, D. K.; Madden, P. A. Mol Phys 1990, 70, 921.

15. Chernyak, V.; Mukamel, S. J Chem Phys 1996, 104, 444-459.

16. Castiglioni, C.; Tommasini, M.; Del Zoppo, M. J Mol Struct 2000, 521, 137-155.

17. Wilson, E. B.; Decius, J. C.; Cross, P. C. Molecular Vibrations; McGraw-Hill: New York, 1955.

18. Del Zoppo, M.; Castiglioni, C.; Zuliani, P.; Zerbi, G. In Handbook of Conducting Polymers; Skotheim, T. A.; Elsenbaumer, R. L.; Reynolds, J., Eds.; Dekker: New York, 1998.

19. Gussoni, M.; Castiglioni, C.; Ramos, M. N.; Rui, M.; Zerbi, G. J Mol Struct 1990, 224, 445.

20. Avery, J. Creation and Annihilation Operators; McGrawHill: New York, 1976.

21. Szabo, A.; Ostlund, N. S. Modern Quantum Chemistry: Introduction to Advanced Electronic Structure Theory; McGraw-Hill: New York, 1989.

22. Pauling, L.; Wilson, E. B. Introduction to Quantum Mechanics; McGraw-Hill: New York, 1935.

23. Feynman, R. P. Phys Rev 1939, 56, 340. 